\title{
Platelets and metastasis revisited: a novel fatty link
}

\author{
Gaorav P. Gupta1 and Joan Massagué1,2
}

${ }^{1}$ Cancer Biology and Genetics Program, and ${ }^{2}$ Howard Hughes Medical Institute, Memorial Sloan-Kettering Cancer Center, New York, New York, USA.

\begin{abstract}
Platelets have long been suspected of having a role in cancer progression and metastasis that has largely been attributed to platelet-mediated enhancement of tumor cell survival, extravasation, and angiogenesis. A study in this issue of the JCI suggests that platelet-derived lysophosphatidic acid is coopted by aggressive breast and ovarian cancer cells as a tumor cell mitogen and promoter of osteolysis during bone metastasis (see the related article beginning on page 1714).
\end{abstract}

\section{The history of platelets and cancer}

Platelets and cancer have been associated clinically since the 1800 s when the French clinician Armand Trousseau diagnosed himself and several other patients with migratory thrombophlebitis (inflammation of vessels due to blood clotting) caused by an occult visceral carcinoma (1). Although Trousseau Syndrome (as it came to be known) is infrequently the initiating clinical presentation of cancer, a majority of advanced cancer patients develop blood platelet abnormalities that indicate a hypercoagulable state (2).

The clinical correlation between platelet dysfunction and cancer progression is supported by the finding that platelets have an essential role in numerous models of experimental metastasis. Depletion of platelets by a variety of mechanisms reduces the number of metastases to lung and bone in both xenograft and syngeneic tumor transplant models (3). Importantly, this phenomenon seems to be quite general, as cancerous cell lines from many tissue types give similar results.

Several mechanisms of platelet action in facilitating metastasis have been proposed (see Figure 1). Aggregates of circulating cancer cells with platelets may protect against immune-mediated pathways of tumor cell clearance (4). In addition, platelets facilitate adhesion of cancer cells to both leukocytes and endothelial cells, which may promote the essential step of extravasation in the metastatic cascade (3). Once the tumor cells have exited the circulation, factors derived

Nonstandard abbreviations used: GPCR, G proteincoupled receptor; LPA, lysophosphatidic acid; $\mathrm{LPA}_{1}$, LPA receptor type 1 .

Conflict of interest: The authors have declared that no conflict of interest exists.

Citation for this article: J. Clin. Invest. 114:1691-1693 (2004). doi:10.1172/JCI200423823. from activated platelets are able to induce neoangiogenesis, thereby enabling growth at the metastatic site (5). In this issue of the JCI, Boucharaba et al. characterize two additional roles of platelets in metastasis: as a direct source of tumor cell mitogens and as an indirect activator of osteoclastic activity in the bone microenvironment (6).

When platelets receive activating signals through their cell surface receptors, they undergo dramatic structural and chemical changes (7). Cytoplasmic granules rapidly fuse with the plasma membrane, releasing a plethora of bioactive compounds into the local periphery and simultaneously exposing activated adhesion receptors to the cell surface $(8,9)$. Concomitant with these membrane events, the cytoskeletal architecture of platelets during activation transforms into a rigid network of actin filaments that is resistant to the shear stresses present in circulation (2). Many of these platelet effectors of hemostasis and thrombosis may have a dual function in promoting the metastasis of tumor cells. Boucharaba et al. now identify platelet-derived lysophosphatidic acid (LPA), a bioactive lipid with growth factor-like signaling properties, as a driving mechanism of bone metastasis (6).

\section{Lysophosphatidic acid: an effector of} platelet-mediated tumor progression

LPA is a structurally simple, water-soluble biolipid, consisting of a phosphoglycerol backbone with only one fatty acyl chain (10). It can be synthesized from multiple enzymatic pathways utilizing either membrane phosphatidic acid or lysophosphatidylcholine as substrates. Upon generation, it is frequently shed into the extracellular environment where it acts in either autocrine or paracrine fashion on a $G$ protein-coupled receptor (GPCR) family of LPA receptors expressed on responsive cells (11). Activation of downstream signaling pathways results in pleotropic effects on normal and transformed cells, including promotion of cellular proliferation, survival, and migration (12). Not surprisingly, aberrant synthesis of LPA has been proposed to enhance tumor growth and progression. Autotaxin, an enigmatic protein whose expression in tumor cells correlates with metastasis, was recently characterized as a lysophospholipase that acts by generating LPA from lysophosphatidylcholine (10). However, the LPA implicated in tumor progression can also be derived from normal host cells, with platelets being a major endogenous source. In fact, levels of LPA reach $1-5 \mu \mathrm{M}$ in serum, yet are several orders of magnitude lower in plasma (10).

The study by Boucharaba et al. (6) is the first to characterize a role for plateletderived LPA as an enhancer of bone metastasis by breast and ovarian cancer cells. The authors primarily utilized a bone-tropic subpopulation of MDA-MB-231 human breast cancer cells engineered to inducibly overexpress LPA receptor type $1\left(\mathrm{LPA}_{1}\right)$ to demonstrate that $\mathrm{LPA}_{1}$ enhances in vivo tumor growth both subcutaneously and in the bone microenvironment. These findings suggest that there must be an endogenous source of LPA mediating the mitogenic effects, either coming from the tumor cells themselves or from a nontransformed host cellular compartment. The authors went on to establish that platelets, and not MDAMB-231 cells, were the source of LPA in this metastasis model. They further demonstrated that MDA-MB-231 cells were able to induce platelet aggregation and activation in vitro, which resulted in liberation of soluble mediators of tumor cell growth (Figure 1). Impressively, the growth-stimulatory activity of the supernatant from activated platelets was dependent on $\mathrm{LPA}_{1}$ and completely abrogated upon pretreatment with LPA-degrading enzymes.

The authors extended these findings in vivo by treating metastasis-harboring animals with Integrilin, a pharmacological 


\section{B Extravasation and growth at secondary site}

III. Adhesion to endothelium, and transmigration

IV. Enhancement of angiogenesis

V. Mitogenic stimulus

VI. Potentiated osteoclastogenesis and osteolysis

\section{A Survival in circulation \\ I. Protection from NK cell- mediated tumor cell death}

II. Resistance to shear stress

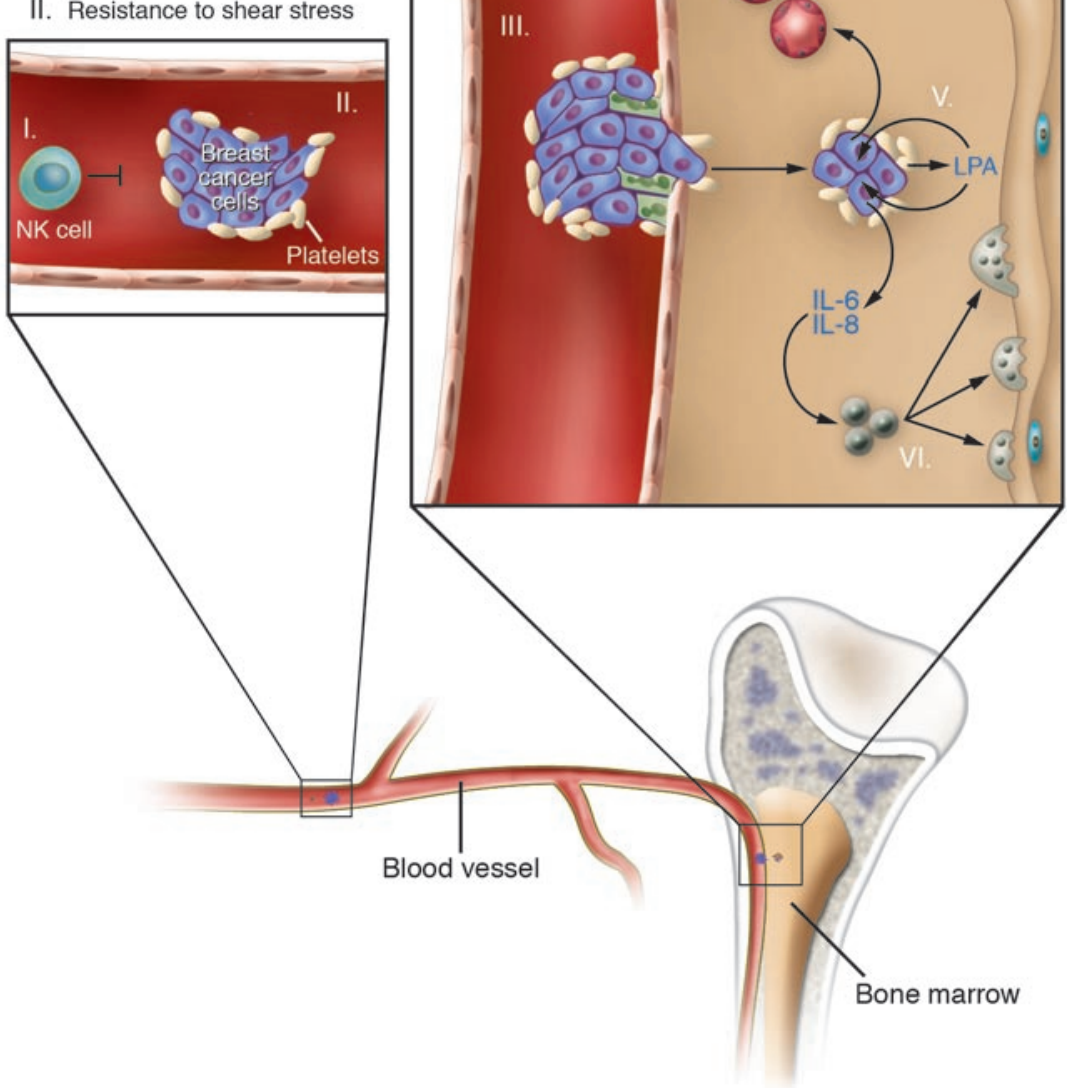

\section{Figure 1}

Platelets facilitate metastasis through multiple mechanisms. (A) Tumor cells induce platelet aggregation and embolus formation, which can enhance survival in the stressful milieu of the circulation. This includes protection from immune-mediated clearance (I) and from shear stresses that are toxic to tumor cells (II). (B) Platelets also form complexes with leukocytes and facilitate adhesion to endothelial cells (III). It is hypothesized that this adhesive cellular aggregate is competent to extravasate into the secondary site of metastasis. Numerous platelet-derived factors enhance angiogenesis (IV), growth, and tissue-specific modulation of the new microenvironment One of these factors is LPA, which acts as a direct tumor cell mitogen (V) and as an enhancer of osteolysis through induction of IL- 6 and IL-8 by cancer cells in the bone marrow (VI).

$\alpha \operatorname{IIb} \beta 3$ integrin antagonist and inhibitor of platelet aggregation (6). Treatment was started after initial bone metastasis formation was detected (approximately 2 weeks after tumor cell inoculation) so as not to interfere with any contribution that platelets may have in early survival and extravasation of metastatic cells. Treated animals exhibited thrombocytopenia, a decrease in circulating LPA plasma levels, and a significant reduction in osteolytic bone metastasis formation. These findings are promising. However, the authors did not interfere specifically with LPA signaling in vivo, leaving open the possibility that this antimetastatic effect could be due to some other LPA-independent pathway. Further work with tumor cells that have not artificially been made hypersensitive to LPA would be necessary to firmly establish
LPA signaling in vivo as a therapeutic target in the treatment of bone metastasis.

Tumor cells that metastasize to bone are not directly able to induce osteolysis. Instead, they often recruit normal host osteoclasts as accomplices in metastasis by releasing osteoclastogenic paracrine factors, such as parathyroid hormone-related protein, IL-8, and IL-11 (13-15). Boucharaba et al. (6) noticed that the enhanced bone metastasis that they observed, owing to $\mathrm{LPA}_{1}$ overexpression, exhibited a disproportionately higher degree of osteolysis. Upon histological examination, they realized that there was more effective osteoclast recruitment and activation by tumor cells with hyperactive LPA signaling. They subsequently demonstrated a potential mechanism explaining this effect: LPA stimulated MDA-MB-231 cells to secrete IL- 6 and IL-8, both potent activators of osteoclastinduced bone resorption (Figure 1).

\section{Platelet modulation as metastasis therapy}

The findings of this study contribute to the already impressive set of preclinical data suggesting that platelet inhibition may slow the rate of tumor progression and metastasis. However, extrapolation of these results into efficacious therapies for cancer patients has proven elusive. Clinical trials of the antiplatelet agents aspirin and heparin have yielded inconclusive, albeit promising, evidence that platelet inhibition may enhance survival of cancer patients $(16,17)$. A primary source of the difficulty in translating these results into effective anticancer therapies is the paramount role that platelets play in maintaining normal hemostasis even in the absence of acute injury. In fact, cancer patients receiving cytotoxic chemotherapy who suffer from bleeding due to platelet toxicity are regularly transfused with large numbers of platelets from healthy donors (2). Is it possible that this life-saving therapy is simultaneously facilitating metastasis of their cancerous cells?

Effective platelet-modulating therapies for metastasis must exhibit specificity for the pathological tumor cell-platelet interaction without abrogating normal platelet function. Platelet-specific integrin inhibitors similar to Integrilin, which was used in the Boucharaba et al. study (6), fall into this category because they inhibit tumor cellinduced platelet aggregation while preserving a partial role for platelets in hemostasis $(18,19)$. Another promising target for inhibition is the adhesive interaction between 
platelet P-selectin and tumor cell sialylated mucins that has been shown to be essential for metastatic spread in mouse models (17). Finally, the findings of Boucharaba et al. lay the groundwork to suggest that inhibition of platelet-derived LPA action on its cognate receptors expressed by tumor cells may be another promising therapeutic target, especially for bone metastasis. The development and clinical testing of this class of specific modulators of platelet function will be necessary before a verdict can be reached regarding the importance of platelets in the progression of disease in cancer patients.

Address correspondence to: Joan Massagué, Memorial Sloan-Kettering Cancer Center, 1275 York Avenue, Box 116, New York, New York 10021, USA. Phone: (212) 639-8975; Fax: (212) 717-3298; E-mail: j-massague@ski.mskcc.org.

1. Rickles, F.R., and Falanga, A. 2001. Molecular basis for the relationship between thrombosis and can- cer. Thromb. Res. 102:V215-V224.

2. Nash, G.F., Turner, L.F., Scully, M.F., and Kakkar, A.K 2002. Platelets and cancer. Lancet Oncol. 3:425-430.

3. Karpatkin, S., and Pearlstein, E. 1981. Role of platelets in tumor cell metastases. Ann. Intern. Med. 95:636-641.

4. Nieswandt, B., Hafner, M., Echtenacher, B., and Mannel, D.N. 1999. Lysis of tumor cells by natural killer cells in mice is impeded by platelets. Cancer Res. 59:1295-1300.

5. Trikha, M., and Nakada, M.T. 2002. Platelets and cancer: implications for antiangiogenic therapy. Semin. Thromb. Hemost. 28:39-44.

6. Boucharaba, A., et al. 2004. Platelet-derived lysophosphatidic acid supports the progression of osteolytic bone metastases in breast cancer. J. Clin. Invest. 114:1714-1725. doi:10.1172/ JCI200422123.

7. Jurasz, P., Alonso-Escolano, D., and Radomski, M.W. 2004. Platelet-cancer interactions: mechanisms and pharmacology of tumour cell-induced platelet aggregation. Br.J. Pharmacol. doi:10.1038/sj.bjp.0706013.

8. FitzGerald, G.A. 1991. Mechanisms of platelet activation: thromboxane $\mathrm{A} 2$ as an amplifying signal for other agonists. Am. J. Cardiol. 68:11B-15B.

9. Brass, L.F. 2003. Thrombin and platelet activation. Chest. 124:18S-25S.

10. Mills, G.B., and Moolenaar, W.H. 2003. The emerging role of lysophosphatidic acid in cancer. Nat. Rev. Cancer. 3:582-591.

11. Ishii, I., Fukushima, N., Ye, X., and Chun, J. 2004.
Lysophospholipid receptors: signaling and biology. Annu. Rev. Biochem. 73:321-354.

12. van Corven, E.J., Groenink, A., Jalink, K., Eichholtz, T., and Moolenaar, W.H. 1989. Lysophosphatidateinduced cell proliferation: identification and dissection of signaling pathways mediated by $G$ proteins. Cell. 59:45-54.

13. Mundy, G.R. 2002. Metastasis to bone: causes, consequences and therapeutic opportunities. Nat. Rev. Cancer. 2:584-593.

14. Yin, J.J., et al. 1999. TGF- $\beta$ signaling blockade inhibits PTHrP secretion by breast cancer cells and bone metastases development. J. Clin. Invest. 103:197-206.

15. Kang, Y., et al. 2003. A multigenic program mediating breast cancer metastasis to bone. Cancer Cell. 3:537-549.

16. Falanga, A. 2004. The effect of anticoagulant drugs on cancer. J. Thromb. Haemost. 2:1263-1265.

17. Varki, N.M., and Varki, A. 2002. Heparin inhibition of selectin-mediated interactions during the hematogenous phase of carcinoma metastasis: rationale for clinical studies in humans. Semin. Thromb. Hemost. 28:53-66.

18. Amirkhosravi, A., et al. 2003. Inhibition of tumor cell-induced platelet aggregation and lung metastasis by the oral GPIIb/IIIa antagonist XV454. Thromb. Haemost. 90:549-554.

19. Bakewell, S.J., et al. 2003. Platelet and osteoclast beta 3 integrins are critical for bone metastasis. Proc. Natl. Acad. Sci. U. S. A. 100:14205-14210.

\section{The Staphylococcus aureus "superbug"}

Timothy J. Foster

Microbiology Department, Moyne Institute of Preventive Medicine, Trinity College, Dublin, Ireland.

\begin{abstract}
There has been some debate about the disease-invoking potential of Staphylococcus aureus strains and whether invasive disease is associated with particularly virulent genotypes, or "superbugs." A study in this issue of the JCI describes the genotyping of a large collection of nonclinical, commensal $S$. aureus strains from healthy individuals in a Dutch population. Extensive study of their genetic relatedness by amplified restriction fragment typing and comparison with strains that are associated with different types of infections revealed that the $S$. aureus population is clonal and that some strains have enhanced virulence (see the related article beginning on page 1732). This is discussed in the context of growing interest in the mechanisms of bacterial colonization, antibiotic resistance, and novel vaccines.
\end{abstract}

\section{Nasal colonization}

Staphylococcus aureus is a common commensal of humans and its primary habitat is the moist squamous epithelium of the anterior nares (1). About $20 \%$ of the population are always colonized with $S$. aureus, $60 \%$ are

Nonstandard abbreviations used: AFLP, amplified fragment length polymorphism; CA-MRSA, community-acquired MRSA; MLST, multilocus sequence typing; MRSA, methicillin-resistant Staphylococcus aureus; PVL; Panton-Valentine leukocidin; WTA, wall teichoic acid.

Conflict of interest: The author has declared that no conflict of interest exists.

Citation for this article: J. Clin. Invest. 114:1693-1696 (2004). doi:10.1172/JCI200423825. intermittent carriers, and 20\% never carry the organism. As there is considerable evidence that carriage is an important risk factor for invasive infection $(1,2)$, it is surprising that so little is known about the bacterial factors that promote colonization of squamous epithelial surfaces and the host factors that determine whether an individual can be colonized or not.

\section{Methicillin-resistant S. aureus}

Healthy individuals have a small but finite risk of contracting an invasive infection caused by $S$. aureus, and this risk is increased among carriers. Hospital patients who are catheterized or who have been treated surgically have a significantly higher rate of infection. In some, but not all, developed countries, many nosocomial infections are caused by $S$. aureus strains that are multiply resistant to antibiotics - known as methicillinresistant Staphylococcus aureus (MRSA) $(3,4)$ - although the acronym MRSA is somewhat misleading because the semisynthetic $\beta$-lactam methicillin is no longer used to treat $S$. aureus infections. In MRSA, the horizontally acquired mecA gene encodes a penicillin-binding protein, PBP2a, which is intrinsically insensitive to methicillin and all $\beta$-lactams that have been developed, including the isoxazoyl penicillins (e.g., oxacillin) that superceded methicillin, in addition to the broad spectrum $\beta$-lactams (third-generation cephalosporins, cefamycins, and carbapenems) that were introduced primarily to treat infections caused by Gram-negative bacteria (4) (Figure 1). In contrast to nosocomial MRSA strains, which are usually multidrug resistant, the recently emerged community-acquired MRSA (CA-MRSA) strains are susceptible to drugs other than $\beta$-lactams (5). 